\title{
Soroprevalência da infecção por Helicobacter pylori em crianças de diferentes níveis sócio-econômicos em Porto Velho, Estado de Rondônia
}

\author{
Seroprevalence of Helicobacter pylori infection among children of different \\ socioeconomic levels in Porto Velho, State of Rondônia
}

Rosely Valéria Rodrigues ${ }^{1}$, Teresa Cristina Corvelo ${ }^{2}$ e Mabel Torres Ferrer ${ }^{3}$

\begin{abstract}
RESUMO
O estudo investigou a soroprevalência de infecção pelo Helicobacter pylori em 200 (subdivididas em 2 grupos) crianças da Cidade de Porto Velho, Rondônia. A prevalência da soropositividade variou consideravelmente de acordo com o nível sócio-econômico, onde $51 \%$ das crianças de baixo nível e $24 \%$ de classe média eram positivas. As características da população infantil relacionadas ao sexo, raça e dieta alimentar não representaram fatores de risco para a aquisição da infecção; porém, a maioria das infectadas pertencia à faixa etária de cinco ou mais anos, independente do nível sócio-econômico. A distribuição fenotípica dos grupos sanguíneos ABO, entre os indivíduos infectados e não infectados, mostrou que a sororeatividade ao Helicobacter pylori foi maior entre as crianças do grupo sanguíneo O, sugerindo que há uma maior susceptibilidade genética destas crianças para a infecção pelo Helicobacter pylori.
\end{abstract}

Palavras-chaves: Helicobacter pylori. Crianças. Nível sócio econômico.

\begin{abstract}
This study investigated the seroprevalence of Helicobacter pylori infection in 200 children in the city of Porto Velho, State of Rondônia, divided in two groups of 100 children. The prevalence of seropositivity varied considerably according to socioeconomic level, such that 51\% of the lower-level children and $24 \%$ of the middle-class children were positive. The characteristics of the child population relating to sex, ethnicity and diet did not represent risk factors for acquiring the infection. However, most of the infected children were in the age group of five years or older, independent of socioeconomic level. The phenotypic distribution of ABO blood groups among the infected and uninfected individuals showed that the seroreactivity to Helicobacter pylori was greater among the children with the O blood type, thus suggesting that these children have greater genetic susceptibility to infection by Helicobacter pylori.
\end{abstract}

Key-words: Helicobacter pylori. Children. Socioeconomic level.

o Helicobacter pylori, é uma bactéria que tem sido muito estudada tanto por sua alta prevalência na população do mundo, quanto pela sua relação com gastrites, úlceras e câncer gástrico ${ }^{17}$.

Muitos estudos ${ }^{6} 9$ têm demonstrado que a infecção pelo Helicobacter pylori inicia-se na infância, especialmente nos paises em desenvolvimento, onde a prevalência atinge $40 \%$ ou mais de crianças na faixa etária dos 10 anos $^{20}$, em contraste com paises desenvolvidos onde a prevalência é bem menor, na faixa de $6 \%$ a $14 \%$ entre jovens e adolescentes. Muitas vezes a bactéria permanece como não patogênica e os portadores persistem assintomáticos ${ }^{4}$ por muitos anos e irão desenvolver quadros gástricos na idade adulta.
$\mathrm{Na}$ infância, o principal fator de risco é a baixa condição socioeconômica, envolvendo inclusive a qualidade da água ingerida, condições precárias de higiene bem como sanitárias ${ }^{11}$, densidade de moradores que compartilham o mesmo quarto da $\operatorname{casa}^{10}$, sendo que muitos outros fatores de risco podem estar envolvidos na alta prevalência do Helicobacter pylori em crianças de países em desenvolvimento.

Existem vários ensaios para diagnosticar a presença da bactéria, entre eles estão: a sorologia, teste histológico, cultura, teste da urease e $\mathrm{PCR}^{8}$. A sorologia é um procedimento não invasivo validado para estudos de prevalência, pois reflete a presença da infecção, uma vez que o contato do organismo com a bactéria desencadeia uma resposta humoral, inicialmente do tipo

1. Departamento de Medicina, Universidade Federal de Rondônia, Porto Velho, Ro 2. Universidade Federal do Pará, Belém, PA. 3. Valfarma Farmácia de Manipulação, Porto Velho, RO.

Endereço para correspondência: Dra. Rosely Valéria Rodrigues. Av. Carlos Gomes 1987, São Cristóvão, 78901-200 Porto Velho, R0.

Tel: 5569 3224-4422; Fax:55 69 3224-3001.

e-mail: valeria@valfarma.com.br

Recebido para publicação em: 14/09/2006

Aceito em: 20/08/2007 
IgA e depois do tipo IgG, que é sistêmica e estável, e só diminui na medida em que a bactéria é erradicada do organismo. Visto que tal processo raramente ocorre de maneira espontânea, na criança esses índices permanecem altos ${ }^{20}$.

O Estado de Rondônia, dentro do contexto nacional não apresenta nenhum estudo sobre as taxas dessa bactéria em sua população. Considerando que o Município de Porto Velho apresenta uma rede precária de esgoto, abastecimento irregular de água tratada, coleta de lixo insuficiente e situa-se na Região Amazônica, onde o manancial hídrico é vasto, e como os estudos sugerem que a água potável é veículo da bactéria ${ }^{22}$, bem como moscas podem funcionar como vetor na veiculação da mesma ${ }^{5}$, evidenciou-se a necessidade de efetuar um estudo desta natureza.

Levando-se também em conta a grande migração ocorrida nos últimos 20 anos para esta região, aonde migrantes de todo o pais vieram em busca de uma nova vida, principalmente em função da atividade de garimpagem, esta região adquiriu a característica de estado em franco desenvolvimento, que ocorreu de forma desordenada, com infra-estrutura precária, característica esta que, em se tratando da infecção por Helicobacter pylori, evidenciou-se a necessidade de efetuar no município um estudo entre classes sociais distintas a fim de observar se a mesma associação - baixo nível social - maior incidência de Helicobacter pylori - ocorre na população de Porto Velho.

Para tanto, foram selecionadas crianças de classes sociais diferentes comparando essas populações entre si, para verificar a prevalência da infecção por Helicobacter pylori, uma vez que a mesma é adquirida predominantemente na infância, até os 10 anos de idade ${ }^{69}$ e que a transmissão oral-oral, gastro-oral e fecal-oral ${ }^{1}$ são as mais prováveis.

O objetivo deste trabalho foi estimar a soroprevalência de Helicobacter pylori em dois grupos sócio- econômicos distintos de crianças no município de Porto Velho, através da resposta sorológica do tipo IgG específica, pela dosagem de anticorpos sanguíneos.

\section{MATERIAL E MÉTODOS}

Casuística. A amostragem foi constituída de 200 crianças na faixa etária de 2 a 13 anos, selecionadas a partir de consultório médico particular e serviço de ambulatório em bairros periféricos. As crianças foram examinadas e encaminhadas para a coleta de material, onde foi preenchido o questionário devidamente autorizado pelos pais a participarem da pesquisa, através do termo de consentimento assinado pelos mesmos. 0 protocolo de estudo foi aprovado pela Comissão de Ética do Núcleo de Medicina Tropical da Universidade Federal do Pará. A condição da pesquisa seguiu os critérios CONEP, conforme a resolução 196/96 do CNBS/MS de acordo com o protocolo CEP/NMT 026/00.

Critérios de inclusão e exclusão. Os critérios de inclusão foram: estar na faixa de idade entre 2 e 13 anos; necessitar de venopunção para exames laboratoriais complementares; aceitar participar do estudo após consentimento livre e esclarecido por parte do responsável e ser morador da área de estudo há mais de 6 meses. Os critérios de exclusão foram: estar de trânsito na comunidade; apresentar alguma patologia que, de acordo com exame médico prévio, não possa participar da pesquisa.

Descrição das amostras. A coleta das amostras foi realizada no período de abril a junho de 2001, na Cidade de Porto Velho, no Estado de Rondônia, Brasil. Compreendendo um total de 200 amostras de sangue, saliva e fezes das crianças, as quais foram posteriormente agrupadas mediante a aplicação do questionário em dois grupos sócio-econômicos distintos, caracterizados por tipo de renda familiar, tipo de moradia e padrão escolar.

Coleta e tratamento das amostras. Foi coletado sangue total, saliva e material fecal para exame parasitológico de fezes.

Saliva: os indivíduos receberam chumaços de algodão para serem umedecidos com saliva. Logo após, o chumaço foi recolhido e acondicionado em frasco estéril, armazenado a $-20^{\circ} \mathrm{C}$. Antes das análises, as amostras foram descongeladas e extraídas dos chumaços para posterior teste.

Sangue: foi coletado por punção venosa periférica aproximadamente $3 \mathrm{ml}$ de sangue de cada criança. 0 sangue foi transferido para frasco estéril contendo heparina como anticoagulante. As amostras foram centrifugadas a 3.000rpm durante 20 minutos. 0 plasma foi estocado a $-20^{\circ} \mathrm{C}$.

Fezes: os responsáveis pelas crianças foram informados para colher as fezes das mesmas sem fazer contato com a água do vaso sanitário.

Técnicas laboratoriais. Dosagem de anticorpos IgG antiHelicobacter pylori específico: o plasma coletado foi utilizado para verificação e dosagem de anticorpos anti-Helicobacter pylori específicos através de ensaios imunoenzimáticos. 0 kit utilizado foi o Immunocard Helicobacter pylori (Meridiam Diagnostics, Inc. USA).

Determinação dos fenótipos ABO e Lewis. Eritrócitos: nos eritrócitos, os fenótipos ABO e Lewis foram tipificados pelos testes de hemaglutinação.

Saliva: na saliva, a caracterização destas referidas especificidades ABH e Lewis foi feita com a técnica dot-blot-ELISA, modificada por Pflug cols ${ }^{21}$.

Estatística. Foram empregados testes estatísticos $\chi^{2}$ para detectar diferenças entre as proporções amostrais dos grupos estudados, 0 programa utilizado foi o BioEstat $2.0^{3}$ A significância estatística foi aceita ao nível $95 \%(\mathrm{P}<0,05$ com poder do teste $>0,8)$.

\section{RESULTADOS}

A prevalência da soropositividade na população investigada variou consideravelmente de acordo com o nível socioeconômico. Enquanto 51\% das crianças de baixo nível sócio econômico eram positivas para a infecção pelo Helicobacter pylori, somente $24 \%$ de nível médio alto apresentaram o mesmo resultado $(\mathrm{p}<0,001)$.

Características da população infantil com relação à infecção pelo Helicobacter pylori. A Tabela 1 indica as características básicas das crianças na amostra estudada, os dados mostram que 52,5\% das crianças eram do sexo masculino 
Tabela 1- Análise univariada entre nível sócio-econômico e outras variáveis da população infantil de Porto Velbo, RO.

\begin{tabular}{|c|c|c|c|c|c|c|c|c|c|}
\hline \multirow[b]{3}{*}{ Características } & \multicolumn{4}{|c|}{ Nível sócio-econômico } & & & & & \\
\hline & \multicolumn{2}{|c|}{ classe baixa } & \multicolumn{2}{|c|}{ classe média alta } & \multicolumn{2}{|c|}{ Total } & \multicolumn{3}{|c|}{ Teste estatístico } \\
\hline & $\mathrm{n}^{0}$ & $\%$ & $\mathrm{n}^{\underline{0}}$ & $\%$ & $\mathrm{n}^{0}$ & $\%$ & $\mathrm{p}$ & $\mathrm{OR}$ & IC $(95 \%)$ \\
\hline Sexo & & & & & & & 0,39 & 0,75 & $0,43-1,31$ \\
\hline masculino & 49 & 46,7 & 56 & 53,3 & 105 & 52,5 & & & \\
\hline feminino & 51 & 53,7 & 44 & 46,3 & 95 & 47,5 & & & \\
\hline Cor & & & & & & & 0,59 & 0,77 & $0,38-1,56$ \\
\hline caucasóide & 78 & 48,8 & 82 & 51,3 & 160 & 80,0 & & & \\
\hline negróide & 22 & 55,0 & 18 & 45,0 & 40 & 20,0 & & & \\
\hline Idade & & & & & & & 0,85 & 1,00 & $0,47-2,09$ \\
\hline$<5$ & 17 & 50,0 & 17 & 50,0 & 34 & 17,0 & & & \\
\hline$\geq 5$ & 83 & 50,0 & 83 & 50,0 & 166 & 83,0 & & & \\
\hline Aleitamento materno & & & & & & & 0,10 & 0,33 & $0,10-1,09$ \\
\hline não & 4 & 26,7 & 11 & 73,3 & 15 & 7,5 & & & \\
\hline $\operatorname{sim}$ & 94 & 51,9 & 89 & 48,1 & 185 & 92,5 & & & \\
\hline Dieta & & & & & & & 0,001 & 2,80 & $1,53-5,13$ \\
\hline incompleta & 47 & 66,2 & 24 & 33,8 & 71 & 35,5 & & & \\
\hline completa & 53 & 41,1 & 76 & 58,9 & 129 & 64,5 & & & \\
\hline
\end{tabular}

$\mathrm{P}<0,05$ com poder de teste $>0,8$. OR: odds ratio

IC(95\%): índice de confiança - 95\%

e cerca de $80 \%$ foram classificadas quanto à cor da pele como caucasóides. A média de idade foi de 7,7 anos (variando de 2 até 13 anos), sendo que entre as crianças selecionadas, 17\% tinham menos do que 5 anos. Em relação ao aleitamento materno das crianças, apenas 15 não tinham recebido leite materno após o nascimento. Por último foi avaliada a dieta ingerida pelas crianças, observando-se que 35,5\% das mesmas a mantinham incompleta, quanto ao conteúdo de fibras, vitaminas e sais minerais, sendo que uma proporção significativa de $66 \%$ destas, estavam agrupadas na classe de baixo nível sócio-econômico.
Considerando a distribuição de freqüência das características individuais acima investigadas, não se observou diferença estatisticamente significativa entre os dois grupos sócioeconômicos, excetuando o fator dieta que mostrou uma dependência em relação ao nível sócio-econômico.

Quando comparamos os sexos feminino e masculino em relação à infecção pelo Helicobacter pylori, não se observou diferenças estatisticamente significantes nos dois diferentes grupos sócio-econômicos, (Tabela 2). Do mesmo modo, a soroprevalência do Helicobacter pylori entre caucasóides e

Tabela 2 - Comparação entre os níveis sócio-econômicos na população infantil de Porto Velho/Ro, segundo as variáveis: sexo, cor, idade, aleitamento materno e dieta.

\begin{tabular}{|c|c|c|c|c|c|c|c|c|}
\hline \multirow[b]{4}{*}{ Categorias } & \multicolumn{6}{|c|}{ Nível sócio-econômico } & \multicolumn{2}{|c|}{ Teste estatístico } \\
\hline & \multirow{2}{*}{\multicolumn{3}{|c|}{$\begin{array}{c}\text { classe baixa } \\
\text { Helicobacter pylorii }\end{array}$}} & \multirow{2}{*}{\multicolumn{3}{|c|}{$\begin{array}{l}\text { classe média-alta } \\
\text { Helicobacter pylori }\end{array}$}} & \multirow{3}{*}{$\begin{array}{c}\chi^{2} \\
\text { Mantel - Haenszel }\end{array}$} & \multirow[b]{3}{*}{$P$} \\
\hline & & & & & & & & \\
\hline & pos & neg & total & pos & neg & total & & \\
\hline Sexo & & & & & & & 0,0005 & 0,98 \\
\hline masculino & 22 & 27 & 49 & 16 & 40 & 56 & & \\
\hline feminino & 29 & 22 & 51 & 8 & 36 & 44 & & \\
\hline Cor & & & & & & & 0,1307 & 0,72 \\
\hline caucasóide & 39 & 39 & 78 & 19 & 63 & 82 & & \\
\hline negróide & 12 & 10 & 22 & 5 & 13 & 18 & & \\
\hline Idade & & & & & & & 6,3399 & 0,01 \\
\hline$<5$ & 6 & 11 & 17 & 0 & 17 & 17 & & \\
\hline$\geq 5$ & 45 & 38 & 83 & 24 & 59 & 83 & & \\
\hline Aleitamento materno & & & & & & & 1,7138 & 0,19 \\
\hline não & 1 & 3 & 4 & 1 & 10 & 11 & & \\
\hline $\operatorname{sim}$ & 50 & 46 & 94 & 23 & 66 & 89 & & \\
\hline Dieta & & & & & & & 0,0055 & 0,94 \\
\hline incompleta & 26 & 21 & 47 & 3 & 21 & 24 & & \\
\hline completa & 25 & 28 & 53 & 21 & 55 & 76 & & \\
\hline Total & 51 & 49 & 100 & 24 & 76 & 100 & & \\
\hline
\end{tabular}


negróides não mostrou diferenças significativas, assim como a infecção pelo $v$ Helicobacter pylori não dependeu do aleitamento materno e dos fatores dietéticos.

As freqüências da sororreatividade ao Helicobacter pylori em crianças com 5 anos ou mais é maior que a de crianças menores de 5 anos com esta infecção em ambos os níveis sócio econômicos, sendo de maior proporção as do grupo de classe sócio-econômico médio-alta.

A associação dos antígenos de grupos sanguíneos ABO, Lewis e estado secretor ABH com a prevalência da infecção pelo Helicobacter pylori. Esta etapa evidencia a distribuição dos fenótipos de grupos sanguíneos ABO, Lewis e estado secretor $\mathrm{ABH}$, que segue as mesmas proporções como descritas em outras populações brasileiras (Tabela 3).

Quando as frequiências destes fenótipos foram analisadas separadamente de acordo com a presença ou ausência da infecção por Helicobacter pylori (Tabela 4), constatou-se que o grupo sanguíneo 0 foi o mais prevalente entre os infectados, sendo que no grupo de crianças Helicobacter pylori positivo sua frequiência foi de $61,3 \%$ enquanto naquelas Helicobacter pylori negativas 0 valor encontrado foi de $45,4 \%(\mathrm{p}<0,05)$.

\begin{tabular}{lcc}
\multicolumn{3}{l}{ Tabela 3- Distribuição dos fenótipos de grupos sanguíneos ABO, Lewis e } \\
\multicolumn{1}{l}{ Estados Secretor ABH na população infantil de Porto Velho, $R$ RO. } \\
\hline Fenótipos & Número & Porcentagem \\
\hline ABO & & \\
O & 99 & 51,0 \\
A & 77 & 39,7 \\
B & 15 & 7,7 \\
AB & 3 & 1,6 \\
Lewis & & \\
Le (a+b-) & 11 & 5,7 \\
Le (a-b+) & 165 & 85,1 \\
Le (a-b-) & 18 & 9,3 \\
Secretor & & \\
secretor & 182 & 93,8 \\
não secretor & 12 & 6,2 \\
\hline Total & 194 & 100,0 \\
\hline
\end{tabular}

Tabela 4 - Expressão dos antígenos de grupos sanguíneos ABO, Lewis e Estado Secretor ABH entre infectados e não infectados na população infantil de Porto Velbo, RO.

\begin{tabular}{|c|c|c|c|c|c|c|}
\hline \multirow[b]{3}{*}{ Grupos sanguíneos } & \multicolumn{4}{|c|}{ Estado Helicobacter pylori } & \multicolumn{2}{|c|}{ Teste estatístico } \\
\hline & \multicolumn{2}{|c|}{ infectados } & \multicolumn{2}{|c|}{$\overline{\text { não infectados }}$} & \multirow[b]{2}{*}{$\chi^{2}$} & \multirow[b]{2}{*}{$P$} \\
\hline & $\mathrm{n}^{0}$ & $\overline{\%}$ & $\mathrm{n}^{0}$ & $\%$ & & \\
\hline$\overline{\mathrm{ABO}}$ & & & & & 4,072 & 0,04 \\
\hline 0 & 46 & 46,0 & 54 & 54 & & \\
\hline $\mathrm{A} / \mathrm{B} / \mathrm{AB}$ & 29 & 30,8 & 65 & 69,1 & & \\
\hline Lewis & & & & & 0,309 & 0,58 \\
\hline $\operatorname{Le}(a-b+)$ & 66 & 39,8 & 100 & 60,2 & & \\
\hline $\operatorname{Le}(a+b-) / \operatorname{Le}(a-b-)$ & 9 & 32,1 & 19 & 67,9 & & \\
\hline Secretor & & & & & 007 & 0,93 \\
\hline secretor & 71 & 39,0 & 111 & 61,0 & & \\
\hline não Secretor & 4 & 33,3 & 8 & 6,7 & & \\
\hline Total & 75 & 119,0 & & & & \\
\hline
\end{tabular}

A análise destes resultados implica na associação entre os grupos sanguíneos e a infecção por Helicobacter pylori, na qual o fenótipo 0 tem uma maior frequiência em direção à infecção pela bactéria.

Como se mostra na Tabela 4 , as distribuições fenotípicas dos sistemas Lewis e estado Secretor ABH não evidenciaram diferenças estatisticamente significantes entre infectados e não infectados pelo Helicobacter pylori.

\section{DISCUSSÃO}

Vários estudos epidemiológicos têm demonstrado evidências de que a infecção primária pela bactéria Helicobacter pylori acontece nos primeiros anos de vida, sendo que a propagação da infecção é facilitada por condições de vida socioeconômicas desfavoráveis. Este estudo corrobora esta hipótese visto que, o grupo de crianças de baixo nível sócio-econômico demonstrou prevalência de 51\% de infectados pela bactéria, enquanto que no grupo de nível sócioeconômico médio-alto, esta taxa foi de $24 \%$.

Como já descrito na literatura ${ }^{15}$, a infecção pela Helicobacter pylori nas crianças deste estudo é igual em ambos os sexos.

Outro ponto importante que deve ser ressaltado é uma maior prevalência da infecção pela Helicobacter pylori em indivíduos negróides ${ }^{10}{ }^{16}$. Esta tendência racial não foi evidenciada neste estudo. Deve-se verificar, porém, se a maior incidência em negros está presente devido a uma susceptibilidade genética ou se é decorrente da condição sócio-econômica, lembrando que em grande parte dos paises em desenvolvimento, a maioria dos negros está distribuída nas classes sócio-econômica mais baixa.

Os dados da literatura mostram que na infância ocorre um rápido aumento da taxa de prevalência da infecção, predominantemente na faixa etária até os 10 anos $^{19}$. Estas informações concordam com as obtidas no estudo em questão onde se observou o aumento da prevalência da infecção acima dos 5 anos, particularmente no nível sócio econômico alto. É importante ressaltar que este perfil da curva de prevalência da infecção, oi evidenciado nas duas classes sócio-econômicas. Assim nos países em desenvolvimento, a prevalência da infecção é mais acentuada após os 5 primeiros anos de vida ${ }^{20}$. Isto pode ser justificado por essa faixa etária representar o início da vida escolar, período onde há um maior compartilhamento dos copos, alimentos, além do próprio conglomerado escolar onde ficam submetidas as crianças desta faixa etária.

0 aleitamento materno nos primeiros seis meses de vida é tido como um fator de proteção para a infecção por Helicobacter pylori. Estudos realizados no Gâmbia, indicam que a amamentação protege as crianças contra a infecção pela Helicobacter pylori através da passagem de anticorpos IgA através do leite ${ }^{23}$. Porém, esta proteção dura enquanto a criança for amamentada. Isto pode explicar porque não foi encontrada neste estudo uma relação entre o tempo de aleitamento materno e a freqüiência da infecção pela Helicobacter pylori, por se tratar de um estudo retrospectivo com relação à prática da amamentação.

A dieta na gênese da Helicobacter pylori associada às alterações gástricas teria óbvia importância prática. As evidências 
demonstram que durante a inflamação, grandes quantidades de radicais livres estão presentes nos tecidos afetados. Estudos que discutem o papel do padrão alimentar pela bactéria indicam que dietas mais saudáveis, ricas em frutas e verduras, conferem proteção na infecção pela Helicobacter pylori ${ }^{7}$. Estas crianças dos 2 níveis sócio-econômicos, o padrão de dieta, completa ou incompleta, diferiram entre estes, contudo não mostrou associação significativa com a infecção pela bactéria. Isto significa que as crianças que ingeriram uma dieta rica em fibras (dieta completa) mostraram a mesma propensão a ter infecção por Helicobacter pylori como as que não tinham este tipo de dieta. Portanto, estes achados sugerem que a dieta não necessariamente pode prevenir a aquisição da infecção por Helicobacter pylori, mas provavelmente atuaria como fator modulador na progressão das patologias gástricas.

Entre os fatores genéticos de importância para um risco maior de infecção por Helicobacter pylori estão os antígenos de grupos ABH e Lewis ${ }^{12}$.

A maioria das bactérias Helicobacter pylori permanece livres no meio gástrico. Contudo, uma parte desta população ataca a mucosa gástrica e pode esporadicamente inclusive entrar a sobreviver dentro das células epiteliais gástricas, desempenhando uma função vital na manutenção da infecçã $0^{13}$, Sendo que vários têm consistentemente demonstrado que Helicobacter pylori utiliza adesinas do tipo lectinas, chamadas Bab A, presentes na sua superfície, para ligar-se aos receptores de grupos sanguíneos na camada de muco e na superfície das células epiteliais ${ }^{24}$.

Alkout cols ${ }^{2}$ demonstraram que a fucose, determinante dos antígenos $\mathrm{H} \mathrm{e} \mathrm{Le} \mathrm{b}^{\mathrm{b}}$, representa um importante receptor expresso na mucosa gastroduodenal aos quais adere 0 Helicobacter pylori $\mathrm{e}$ os fenótipos 0 e Le $(\mathrm{a}-\mathrm{b}+)$ expressam uma grande quantidade destes antígenos fucosilados em comparação com outros grupos. Como estes antígenos, não são modificados para antígenos A e/ou B no grupo 0 , sua expressão elevada seria relevante na relação e entre este grupo sanguíneo, a infecção por Helicobacter pylori e suas patologias gástricas, independente dos fenótipos Lewis e a condição secretor ou não-secretor das substâncias ABH ${ }^{18}$. Além do que, em crianças e adolescentes a expressão de Le $\mathrm{L}^{\mathrm{b}}$, e subseqüentemente a ligação do Helicobacter pylori é mais baixa do que em adultos.

Neste estudo, a distribuição dos fenótipos Lewis e Estado Secretor ABH não mostraram diferenças significativas entre as crianças infectadas e não infectadas pelo Helicobacter pylori. Contudo, foi detectada uma forte associação entre o grupo sanguíneo 0 e a infecção causada pelo Helicobacter pylori, o que é reforçada pelos dados obtidos de outros estudos, como 0 de Lin cols $^{14}$ que constataram uma elevada frequiência da infecção como esta bactéria em 90,3\% dos pacientes do grupo 0 acometidos com doenças gastroduodenais. Do mesmo modo, Mattos cols ${ }^{18}$ também observaram que a prevalência do fenótipo de grupo sanguíneo 0 era significativamente elevada $(58,8 \%)$ entre os pacientes com úlcera duodenal péptica, duodenal gástrica $\mathrm{e}$ infectado por Helicobacter pylori.

Em síntese, estas observações apóiam a hipótese de que as pessoas do tipo sanguíneo 0 possuem maior susceptibilidade a infecção por Helicobacter pylori.

\section{REFERÊNCIAS}

1. Akamatsu T, Tabata K, Hironga M, Kawahamj H, Uyeda M. Transmission of Helicobacter pylor i infection via flexible fiber optic endoscopy. American Journal Infection Control 24: 396-401, 1996

2. Alkout AM, Blackwell CC, Weir DM, Poxton IR, Elton RA, LumanW, Palmer K Isolation of a cell surface component of Helicobacter pylori that binds H type 2, Lewis $^{\mathrm{a}}$ and Lewis ${ }^{\mathrm{b}}$ antigens. Gastroenterology 112: 1179-1187, 1997.

3. Ayres M, Ayres JR, Ayres DL, Santos AS. Bio estat 2.0: aplicações estatísticas nas áreas das ciências biológicas e médicas. Belém: Sociedade Civil Mamirauá. Brasilia: CNPq, 2000.

4. Blecker U, Hauser B, Lanciers S, Peeters S, Suys B, Vandenplas Y. The prevalence of Helicobacter pylori positive serology in asymptomatic children. European Journal Pediatric 134: 32-38, 1996.

5. Brenner H, Bode G, Adler G, Rothenbacher D. Does maternal smoking hinder mother child transmission of Helicobacter pylori infection? Epidemiology 11: 71-75, 2000.

6. Brenner H, Rothenbacher D, Bode G, Dieudonné J, Adler G. Active infection with Helicobacter pylori in healthy couples. Epidemiololgy Infectology 122: 91-95, 1999.

7. Brown LM. Helicobacter pylori: epidemiology and routes of transmission. Epidemiologic Reviews 22: 283-297, 2000.

8. Dunn BE, Cohen H, Blaser MJ. Helicobacter pylori virulence factor in gnotobiotic piglets. Infection and Immunity 57: 1119-1125, 1997.

9. Gastron MM, Tinderg Y, Blennow M. Soroepidemiology of Helicobacter pylori infection in a cohort of children monitored from 6 months to 11 years of age. Journal of Clinical Microbiology 16: 247-251, 1997.

10. Graham D, Malaty HM, Evans DG; Evans DJJ, Klein PD, Adam E. Epidemiology of Helicobacter pylori in asymptomatic population in the United States. Gastroenterology 100: 1495-1501, 1991.

11. Handt LK, Fox JG, Dewhist FE, Fraser GJ, Paster BJ, Yan LL. Helicobacter pylori isolated from the domestic cat: public health implications. Infection and Immunity 62: $2367-2374,1994$

12. Ilver D, Arnqvist A, Ögren J, Frick IM, Kersulyte D, Incecik ET, Berg DE, Covacci A, Engstrand L, Borén T. Helicobacter pylori adhesion fucosilated histo-blood antigens revelead by retagging. Science 279: 373-377, 1998.

13. Kirschner DE, Balser M. The dynamics of Helicobacter pylori infection of the human stomach. Journal of Theorical Biology 176: 281-290, 1995.

14. Lin CW, Chang YS, Wu SC, Cheng KS. Helicobacter pylori in gastric biopsies of Taiwanese patients with gastroduodenal disease. Japanese Journal of Medical Science Biology 51: 13, 1998

15. Malaty HM, El-Kasabany A, Graham DY, Miller CC, Reddy SG, Srinivasan SR, Yamaoka Y, Berenson GS. Age at acquisition of Helicobacter pylori infection: a follow-up study from infancy to adulthood. The Lancet 16: 931-935, 2002.

16. Malaty HM, Evans DG, Evans Jr DJ, Graham DY. Helicobacter pylori in Hispanics: comparison with blacks and whites of similar age and socioeconomic class. Gastroenterology 103: 813-816, 1992.

17. Marshall BJ, Warren JR. Unidentified curve bacillus on gastric epithelium in active chronic gastritis. The Lancet 1: 1273-1275, 1983.

18. Mattos LM, Cintra JR, Sanches FE, Silva RCMA, Ruiz MA, Moreira HM. ABO, Lewis, Secretor phenotypes in patients infected or uninfected by Helicobacter pylori bacillus. São Paulo Medical Journal 120: 55-58, 2002.

19. Mitchell HM, Li YY, Hu PJ, Liv Q, Chen M, Du G. Epidemiology of Helicobacter pylori in southern China: identification of early childhood as the critical period for acquisition. Journal of Infectious Disease 166: 149-153, 1992.

20. Patel P, Mendall M, Khulusi S. Helicobacter pylori infection in children: risk factors and effect on growth. British Medical Journal 309: 119-123, 1994.

21. Pflug W, Bässler G, Eberspacher B. ABO and Lewis typing secretion stain on nitrocellulose membranes using a new dot-blot-ELISA technique. Forensic Science International 43: 171-182, 1989.

22. Shauer D B, Handwerker L, Correa P, Fox J. Detection of Helicobacter pylori in drinking water using PCR amplification. European Helicobacter pylori Study Group $8^{\text {th }}$ International Conference, 1995.

23. Thomas JE, Austin S, Dale A, McLean P, Harding M, Coward WA, Weaver LT. Protection by human milk IgA against Helicobacter pylori infection in infancy. The Lancet 342: 121, 1993.

24. Wadstron T, Hirmo S, Nilsson B. Biochemical aspects of $H$. pylori adhesion Journal of Physiology and Pharmacology 48: 325-331, 1997. 